\title{
Analyse d'une démarche de résolution de problèmes environnementaux en République de Guinée
}

Marc Boutet, Amélie Gadbois, Ghislain Samson, Mamadou Bhoye BAH et Kadiatou Nour Diallo

\section{(2) OpenEdition}

1 Journals

Édition électronique

URL : https://journals.openedition.org/ere/787

DOI : $10.4000 /$ ere. 787

ISSN : 2561-2271

Éditeur

Centr'ERE

Édition imprimée

Date de publication : 20 décembre 2016

ISSN : 1373-9689

Référence électronique

Marc Boutet, Amélie Gadbois, Ghislain Samson, Mamadou Bhoye BAH et Kadiatou Nour Diallo,

"Analyse d'une démarche de résolution de problèmes environnementaux en République de Guinée », Éducation relative à l'environnement [En ligne], Volume 13 - 2 | 2016, mis en ligne le 20 décembre 2016, consulté le 21 septembre 2021. URL : http://journals.openedition.org/ere/787 ; DOI : https://doi.org/ $10.4000 /$ ere. 787

Ce document a été généré automatiquement le 21 septembre 2021.

La revue Éducation relative à l'environnement est mise à disposition selon les termes de la Licence Creative Commons Attribution - Pas d'Utilisation Commerciale 4.0 International. 


\title{
Analyse d'une démarche de résolution de problèmes environnementaux en République de Guinée
}

\author{
Marc Boutet, Amélie Gadbois, Ghislain Samson, Mamadou Bhoye BAH et \\ Kadiatou Nour Diallo
}

1 La démarche présentée et analysée dans cet article a été réalisée en République de Guinée. Elle constitue un volet d'un projet international intitulé Vers la résolution créative de problèmes environnementaux sous la responsabilité du Groupe de recherche Littoral et vie de l'Université de Moncton. Comme nous l'avons fait en Guinée, d'autres équipes de recherche du Brésil, de la Colombie, de la Roumanie et du NouveauBrunswick ont mis en place et décrit l'impact de démarches de résolution de problèmes environnementaux vécues par des jeunes de divers groupes d'âge, du début du primaire jusqu'à la fin du secondaire. Les objectifs communs étaient premièrement, de décrire comment les élèves posent (énoncent, représentent) des problèmes environnementaux, pendant et à la suite de l'étude de ceux-ci et deuxièmement, d'expérimenter diverses stratégies pédagogiques ayant pour but d'améliorer les compétences des élèves à poser un problème environnemental de façon à lui trouver des solutions efficaces et créatives. Cet article ne concerne que le volet Guinée.

2 Nous spécifions d'abord le contexte, la problématique et les objectifs de cette recherche. Par la suite, nous situons conceptuellement la démarche éducative proposée aux jeunes par rapport aux approches par problèmes en éducation relative à l'environnement et au développement durable (EREDD). Les choix méthodologiques sont ensuite précisés et justifiés avant que ne soient présentés et interprétés les résultats. 


\section{Le contexte de la recherche}

3 La République de Guinée est un petit pays de l'Afrique de l'Ouest d'une superficie de $245857 \mathrm{~km}^{2}$, dont la capitale Conakry est située sur la côte Atlantique (Mission économique de Conakry, 2004). Malgré des ressources minérales et agricoles importantes, l'abondance en eau et une capacité hydroélectrique considérable, la Guinée demeure une nation essentiellement " sous-développée » (CIA, 2009). L'exode rural important dans le pays a eu pour effet de concentrer la majeure partie de la population dans les grandes villes et particulièrement dans la capitale, Conakry. Malgré la richesse du pays en réserves d'eau douce, Camara (2004) souligne que l'approvisionnement en eau potable y est un enjeu important.

4 Notre dispositif de recherche en Guinée s'est appuyé sur un partenariat déjà existant entre la Faculté d'éducation de l'Université de Sherbrooke et l'École normale des professeurs de l'enseignement technique et professionnel de la République de Guinée, dans le cadre duquel une formation pédagogique a été offerte par la faculté aux professeures et professeurs de l'École normale. Le chercheur principal de l'étude qui fait l'objet de cet article a été responsable de ce programme de formation pendant toute sa durée (Boutet, 2002).

5 Une professeure guinéenne formée à la maîtrise et ayant consacré son essai à l'éducation relative à l'environnement et au développement durable (EREDD), a joué un rôle important dans ce nouveau projet axé sur la résolution de problèmes, agissant à titre de coordonnatrice des activités de formation et de recherche sur le terrain, en relation étroite avec l'équipe de chercheurs. Elle était appuyée par un comité d'experts dont l'un des membres est professeur au Centre d'éducation et de recherche en environnement de l'Université de Conakry et a d'ailleurs, comme la coordonnatrice, contribué à la rédaction de cet article.

\section{La problématique et les objectifs}

6 Nous avons l'habitude d'utiliser l'expression Éducation relative à l'environnement et au développement durable (EREDD) pour désigner le domaine éducatif dans lequel nous nous situons. En adoptant cette formulation, nous souhaitons dépasser la traditionnelle opposition entre environnement et développement, pour orienter les efforts éducatifs vers un futur à construire sur d'autres bases que celles qui ont conduit aux situations actuelles de déséquilibres écologiques, d'injustices sociales et d'inégalités économiques (Éducation, formation professionnelle et jeunesse Manitoba, 2001). L'idée que la redéfinition de la relation entre l'être humain et son environnement soit l'objet central de ce domaine éducatif (peu importe comment on le désigne) fait par ailleurs l'objet d'un large consensus (Sauvé, 1997). L'une des dimensions reconnues de cette redéfinition est la capacité de résolution des problèmes environnementaux créés par l'être humain.

7 Les problèmes environnementaux, dont ceux portant sur la qualité de l'eau, sont des problèmes complexes qui prennent leur source dans les pratiques sociales et les valeurs supportées par les individus et les sociétés (Bardwell et coll., 1994). En raison de leur complexité, les savoirs de multiples disciplines et de divers types doivent être convoqués pour les cerner de façon complète. Quant à la prise de décision pour 
déterminer les actions appropriées pour les résoudre, elle requiert l'interaction de divers protagonistes n'ayant pas les mêmes intérêts, ce qui fait que les solutions apportées sont généralement basées sur des valeurs et controversées (Bardwell et coll., 1994). Le traitement des problèmes environnementaux est donc souvent caractérisé par l'incertitude et leur résolution ne se situe pas uniquement à un niveau technique. Cela nécessite aussi des compétences réflexives complexes, le développement de la pensée critique (UNESCO-PNUE, 1978) et une bonne dose de créativité (Treffinger, 1995).

8 Face à de telles exigences, on peut se demander si les jeunes du primaire et du secondaire possèdent les capacités nécessaires pour mener des démarches de résolution de problèmes environnementaux. Peu de recherches ont été réalisées sur le sujet, tant en didactique des sciences qu'en éducation relative à l'environnement (Wilsson, 1995). On connaît peu de choses sur la manière dont les élèves peuvent devenir des agents de résolution de problèmes (Wheatley, 1995). Certaines initiatives ont déjà été entreprises dans le domaine que nous nommons EREDD (Hart, 1997; Pruneau et coll., 1999; Chawla, 2002; Driskell, 2002), semblant démontrer chez les jeunes l'intérêt et les capacités nécessaires à leur engagement pour le développement durable de leur communauté. Cependant, Pruneau et coll. (2009) précisent qu'il n'existe pas de travaux visant à décrire clairement les capacités des jeunes à poser et à résoudre un problème environnemental.

9 Notre recherche s'intéresse donc aux capacités des jeunes de poursuivre et de compléter une démarche de résolution de problèmes environnementaux. Plus spécifiquement, nous avons cherché à déterminer si leur participation à une démarche de résolution de problèmes leur permettait de mobiliser leurs connaissances, de susciter leur intérêt pour la protection de l'environnement et le développement durable, et de développer leur désir de passer à l'action. Il s'agit là en effet de trois composantes d'un engagement citoyen pour l'environnement reconnues depuis longtemps (Unesco-PNUE, 1978).

\section{Le cadre conceptuel}

10 L'approche par problèmes (APP) serait associée aux travaux de Barrows (1986) qui met au point cette approche en se basant sur deux formules pédagogiques déjà existantes : la méthode des cas de l'Université Harvard et l'apprentissage par la découverte de Bruner (1971, in Ménard, 2007, p. 92).

11 L'analyse de la documentation scientifique montre que l'approche par problèmes peut prendre différentes formes (Tremblay, 2009) sur un continuum allant d'une pédagogie fermée à une pédagogie ouverte. Tantôt cette approche renvoie aux problèmes d'application (par exemple, appliquer une formule connue pour résoudre un problème donné), tantôt à des situations de découverte dirigées (problème formulé par l'enseignant qui guide ensuite l'élève par un ensemble de questions, d'observations ou de documents choisis) et tantôt à des situations-problèmes ouvertes dont l'exploration implique un plus grand engagement de l'élève. Sans négliger l'importance des deux premières modalités d'opérationnalisation de l'approche par problèmes, c'est ce dernier type d'approche, centré sur la démarche des élèves et leur engagement dans l'investigation et dans la structuration des savoirs, qui nous intéresse particulièrement.

C'est donc la définition de l'APP proposée par Galaise (2001, p. 141) que nous retenons: 
L'apprentissage par problèmes est une approche pédagogique où le problème sert de motivation à l'apprentissage. Les étudiants sont confrontés au problème sans étude ou présentation préalable, ni de la matière ni du problème, et ont alors à déterminer les connaissances qu'ils possèdent et celles qui leur manquent pour solutionner le problème. L'acquisition de nouvelles connaissances se fait entre chaque rencontre de travail sur le problème, en fonction des besoins individuels. En APP, l'accent est mis sur l'analyse du problème et non sur la solution.

13 Les étapes constituant le processus de résolution de problèmes varient quelque peu entre les différents auteurs s'intéressant au sujet. Les modèles proposés comptent habituellement entre quatre et huit étapes. Le canevas présenté ici découle d'une mise en commun de démarches proposées par différents auteurs (Bardwell, 1991; Ouellet, 1994; Pólya, 1971; Proulx, 1999; Sauvé et coll., 2001) :

- Étape 1 : Repérer le problème ;

- Étape 2 : Analyser la situation problème ;

- Étape 3 : Poser le problème ;

- Étape 4 : Rechercher des solutions ;

- Étape 5 : Évaluer les diverses solutions et choisir la solution optimale ;

- Étape 6 : Mettre au point un plan d'action;

- Étape 7 : Le mettre en œuvre ;

- Étape 8 : Évaluer et faire le suivi.

\section{Dispositif méthodologique}

14 Le projet international dans lequel s'inscrit notre recherche en Guinée est une étude multi-cas dont l'avantage, selon Miles et Huberman (1991), est son potentiel de généralisation. En Guinée, comme ce fut fait également dans les autres pays, nous avons choisi d'adapter le dispositif de recherche au contexte naturel pour explorer de façon inductive (Mucchielli, 1996) le phénomène qui nous intéressait. En ce sens, notre étude du cas peut être considérée de type descriptif, au sens où l'entend Merriam (1988), c'est-à-dire que nous visions une description détaillée de l'objet d'études, permettant certaines interprétations.

L'expérimentation a eu lieu dans trois classes d'écoles différentes de la capitale, Conakry, durant les mois de janvier à juin $2007^{\text {i }}$. L'une d'elles regroupait vingt-deux élèves de $6^{\mathrm{e}}$ année du primaire (11-12 ans) de l'école privée Les Élites. Un deuxième groupe était formé de dix-neuf élèves de dernière année du secondaire (16-17 ans) en option sciences expérimentales, du groupe scolaire privé Hamdallaye. Les trente élèves $\mathrm{du}$ troisième groupe étaient en $3^{\mathrm{ème}}$ année de formation professionnelle en plomberie (jeunes adultes) au Centre de formation professionnelle (CFP) public de Donka. Tous ces jeunes ont été invités à explorer la problématique de l'eau dans leur milieu.

16 Le scénario pédagogique présenté au Tableau 1 s'est déroulé sur dix ou quinze semaines, selon que les élèves choisissaient ou non de passer à l'action. Ce scénario a d'abord été planifié par l'équipe de recherche puis adapté au contexte de chaque classe avec les enseignants. À tous moments, les élèves étaient encouragés à inscrire dans un journal réflexif leurs préoccupations et leurs sentiments face au projet, leurs questions et leurs réponses aux questions posées en classe. Par ailleurs, les assistants de recherche présents sur place ont consigné les discussions et les observations faites en classe, en plus de colliger les réponses aux questionnaires complétés par les apprenants au fil du déroulement du scénario. 
Tableau 1. Résumé du scénario

\begin{tabular}{|l|l|}
\hline Semaine 1 & Amorce du projet : susciter l'intérêt et repérer des problèmes. \\
\hline Semaine 2 & Sortie d'exploration du milieu immédiat \\
\hline Semaine 3 & Étude des problématiques environnementales reliées à l'eau \\
\hline Semaine 4 & Observations \\
\hline Semaine 5 & Choix d'une problématique par équipe \\
\hline Semaine 6 & Description opérationnelle des problématiques \\
\hline Semaine 7 & Formulation de solutions possibles \\
\hline Semaine 8 & Évaluation et choix de solutions à mettre en œuvre \\
\hline Semaine 9 & Planification de l'action \\
\hline Semaine 10 & $\begin{array}{l}\text { Décision de passer à l'action } \\
\text { Sinon, bilan de la démarche vécue }\end{array}$ \\
\hline Semaines 11-14 & Si oui, action et régulation de l'action \\
\hline Semaine 15 & Évaluation de l'action et bilan de la démarche \\
\hline
\end{tabular}

Les semaines 1 à 6 ont permis aux élèves d'élaborer leur conception du problème afin de pouvoir mieux le poser. Lors de la semaine 2, les jeunes ont fait une sortie d'exploration multisensorielle afin de repérer directement les problèmes. Ils ont effectué une nouvelle sortie pendant la semaine 4, pour observer plus spécifiquement les problématiques liées à la pollution de l'eau et tenter d'en identifier les causes. La démarche s'est poursuivie de la semaine 7 à la semaine 10 par la formulation de solutions puis d'un plan d'action en équipe. Les trois classes ayant décidé de passer à l'action, les semaines suivantes furent consacrées à la mise en œuvre du plan retenu et à l'évaluation de l'efficacité de l'action.

\section{Description des données recueillies}

Malgré certaines difficultés liées au contexte, nous avons quand même pu recueillir suffisamment d'informations pour procéder à des analyses significatives. Notre corpus de données est constitué des éléments suivants :

- Source 1 : les notes prises pendant les activités vécues avec les élèves chaque semaine par les trois assistants de recherche sur le terrain. Celles-ci rendent compte du déroulement réel de la démarche de résolution de problème et des réactions des élèves exprimées dans leurs journaux réflexifs ou oralement. 
- Source 2 : dessins et affiches. Au total 38 dessins et affiches réalisés en équipes ont pu être recueillis, représentant leur conception du problème à diverses étapes de la démarche :

- 12 productions réalisées à la semaine 1 en réponse à la question : Connaissez-vous des sources de pollution de l'environnement et leurs conséquences?

- 13 autres réalisées à la semaine 3 pour illustrer la compréhension qu'avaient les élèves des problèmes reliés à l'eau ;

- 13 illustrations réalisées à la semaine 6 pour représenter le problème que l'équipe se propose de résoudre.

- Source 3 : différents autres documents produits par les apprenants durant la démarche de résolution de problèmes, tels que des fiches d'observation, des outils de planification d'un plan d'action et des textes de sensibilisation (destinés à leur communauté) écrits pendant les semaines de régulation de l'action.

- Source 4 : réponses des élèves à deux questionnaires. Le premier a été distribué à la semaine 6 et portait sur l'évolution de leur représentation des problèmes reliés à l'eau. Le deuxième, un questionnaire bilan sur la démarche vécue, a été distribué à la semaine 15 après que les élèves aient passé à l'action; nous avons pu recueillir le deuxième questionnaire rempli par 45 des 61 élèves.

\section{Méthode d'analyse des données}

Notre analyse des données s'est faite sous le mode de l'« induction analytique » (Deslauriers, 1997, p. 293). À l'intérieur des frontières que nous avions établies par la mise en œuvre d'un scénario pédagogique, nous avons voulu examiner le processus et les résultats de l'expérimentation d'une démarche de résolution de problèmes environnementaux en contexte scolaire africain. Cependant, nous ne prétendons nullement « en déduire, si possible, une explication universelle (Deslauriers, 1997, p. 296). Même l'ensemble des données recueillies et des analyses faites pour tous les volets du projet international ne sauraient suffire à atteindre une telle universalité au regard des objectifs communs poursuivis.

Afin que notre étude de cas puisse s'inscrire dans un corpus de recherches pertinentes par rapport à l'objet ciblé, nous avons appliqué les critères de scientificité suivants, parmi l'ensemble de ceux reconnus en approche qualitative (Laperrière, 1997) :

- la prise en compte du positionnement particulier à la fois des sujets de l'étude et des assistants de recherche sur le terrain;

- la présence prolongée de ces derniers à proximité de la démarche;

- l'élaboration de descriptions en profondeur et la poursuite d'un consensus intersubjectif entre les assistants guinéens ainsi qu'avec les enseignants lors de la rédaction conjointe des rapports hebdomadaires;

- une codification précise des observations empiriques, faite par les deux assistants de recherche québécois sur l'ensemble des données codifiables;

- la confrontation systématique des éléments d'interprétation à l'ensemble des données.

Le procédé utilisé pour satisfaire à ce dernier critère a été de tracer un portrait des données de sources 1,2 et 3 dont nous disposions pour chaque semaine du scénario pédagogique pour chaque école. Puis, nous avons fait ressortir les principaux éléments dans chaque école, pour chaque activité du scénario au sujet de laquelle nous avions des données. Nous présentons au Tableau 2 un bref extrait des nombreux tableaux que nous avons ainsi élaborés. 
Tableau 2. Exemple d'analyse de données pour deux écoles

\begin{tabular}{|c|c|c|}
\hline $\begin{array}{l}\text { Semaine } 1 \\
\text { Dessins } \\
\text { sur les sources } \\
\text { de pollution }\end{array}$ & $\begin{array}{l}\text { École les Élites }(\mathrm{n}=22) \\
\text { Tous les dessins évoquent la pollution de } \\
\text { l'eau, de la terre et de l'air par des activités } \\
\text { anthropiques. } \\
\text { Le mot pollution revient } 14 \text { fois, et dans } 3 \\
\text { des } 4 \text { dessins on retrouve un cours d'eau. } \\
\text { Un dessin évoque la contamination par } \\
\text { défécation. } \\
\text { Deux dessins font référence à la fumée. }\end{array}$ & $\begin{array}{l}\text { École Hamdallaye (n=19) } \\
\text { Sur } 3 \text { des } 4 \text { dessins, on retrouve un } \\
\text { cours d'eau (un dessin s'intitule la } \\
\text { pollution de l'eau). } \\
2 \text { dessins sur } 4 \text { évoquent la } \\
\text { contamination par défécation. } \\
2 \text { dessins font référence à la fumée. } \\
1 \text { dessin met l'accent sur un cadavre } \\
\text { d'animal et les feux de brousse. }\end{array}$ \\
\hline $\begin{array}{l}\text { Semaine 1 } \\
\text { Expression } \\
\text { personnelle des } \\
\text { élèves }\end{array}$ & $\begin{array}{l}\text { Ce qu'ils aiment: Une maison et un } \\
\text { environnement propres. } \\
\text { Ce qu'ils n'aiment pas: les saletés, les eaux } \\
\text { usées, la poussière, la coupe abusive du } \\
\text { bois, les feux de brousse, les déchets jetés } \\
\text { partout et qui causent des maladies. }\end{array}$ & $\begin{array}{l}\text { Ce qu'ils aiment : la faune et la flore, } \\
\text { la propreté de leur milieu, de l'eau } \\
\text { et de l'air; } \\
\text { Ce qu'ils n'aiment pas : la présence } \\
\text { des ordures partout, les feux de } \\
\text { brousse, la déforestation, les engins } \\
\text { et les ordures fumant. }\end{array}$ \\
\hline
\end{tabular}

Les données recueillies à l'aide des deux questionnaires (source 4) ont fait l'objet d'une compilation particulière dans des tableaux permettant de comparer les réponses d'une école à l'autre. L'utilisation de fréquences s'est avérée nécessaire étant donné que le nombre de répondants variait d'une réponse à l'autre. Sur cette base, un bilan global de même qu'un bilan par école de la démarche ont pu être établis.

Nous avons également analysé les données, pour chaque école,

- en fonction des trois grandes étapes de la démarche de résolution de problèmes: poser le problème, planifier l'action, mettre en ouvre et évaluer l'action;

- et au regard des trois objectifs poursuivis, soit le développement de la capacité de mobiliser des connaissances, de l'intérêt pour l'environnement et le développement durable, du désir de passer à l'action

\section{Présentation et discussion des principaux résultats}

L'espace de cet article ne permettant pas d'y intégrer toute l'information contenue dans les différents tableaux que nous avons produits à la suite des analyses, nous avons donc choisi de ne présenter qu'un extrait de l'un des tableaux pour illustrer notre méthode. C'est dans le texte qui suit que seront présentés et discutés les principaux résultats, d'abord en lien avec les étapes de la démarche de résolution de problèmes, puis en lien avec les objectifs de formation poursuivis.

\section{Étape Poser le problème}

Le tableau qui suit (Tableau 3) est un extrait d'un tableau synthèse que nous avons réalisé en lien avec l'étape Poser le problème. Nous l'intégrons ici pour montrer comment 
nous avons regroupé et examiné les données au regard de l'atteinte des objectifs poursuivis.

Tableau 3. Points communs aux trois écoles relativement à l'atteinte des objectifs pour l'étape Poser le problème - Extraits

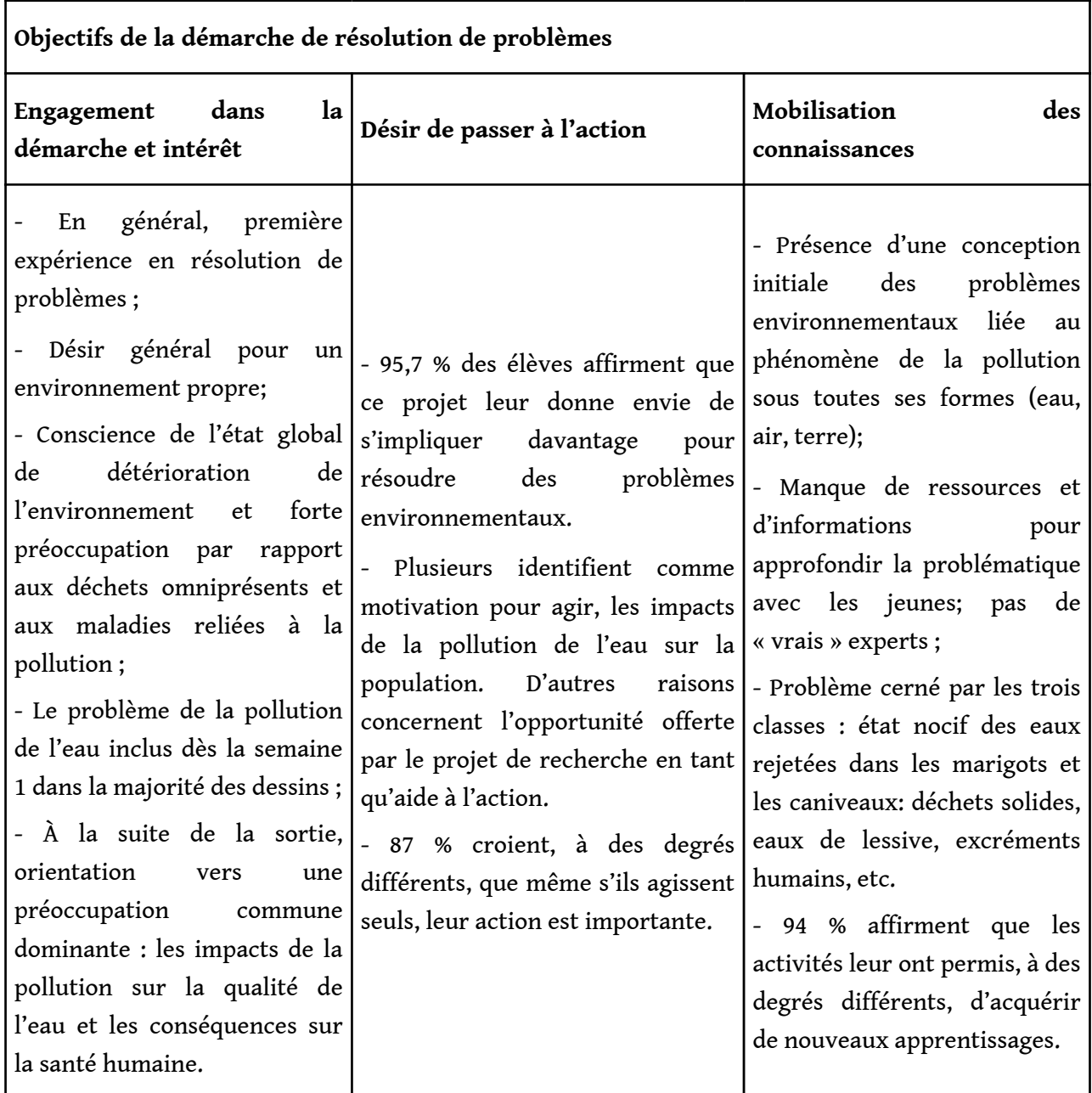

Nous avons noté que la plupart des conceptions initiales étaient liées au phénomène de la pollution sous différentes formes (eau, air, terre) et que, déjà à cette étape du processus, les apprenants avaient une certaine conscience de l'importance particulière des problématiques reliées à l'eau. En effet, notre analyse, qui tentait d'identifier les sources de pollution de l'environnement et leurs conséquences, a montré que la majorité des dessins exécutés par les élèves durant cette étape font référence à la pollution de l'eau. L'analyse révèle cependant que leurs conceptions du phénomène demeurent imprécises voire inexactes. Comme Haulsein et Smith (1994) l'expliquent, les novices n'organisent pas leurs connaissances comme le feraient des experts. Ainsi quand vient le temps d'utiliser les connaissances utiles à la résolution du problème qui les préoccupent, celles-ci sont moins maîtrisées et intégrées. Par ailleurs, en contexte guinéen, les ressources disponibles afin d'aider les apprenants à enrichir et à approfondir leurs connaissances étaient limitées. De plus, le scénario général proposé par Pruneau (2006) prévoyait la présence de scientifiques experts, afin d'aider les apprenants à analyser le problème en profondeur, ce qui n'a pu être possible pour les 
jeunes guinéens ${ }^{\mathrm{ii}}$. C'est ainsi qu'on note, après 6 semaines, que seulement $55 \%$ des jeunes affirment se sentir outillés pour bien poser et décrire le problème relié à l'eau qu'ils ont choisi.

Signalons qu'à l'amorce du projet, les apprenants paraissaient déjà démontrer, un intérêt évident pour la problématique de la qualité de l'eau, qui est omniprésente en Guinée. Cet intérêt a semblé s'être confirmé en réponse à la sortie d'exploration vécue par les jeunes et se reflète dans leurs réponses au questionnaire de la semaine 6. À l'occasion de la sortie dans leur milieu, les jeunes se sont en effet orientés vers une préoccupation commune dominante, relative aux impacts de la pollution sur la qualité de l'eau et aux conséquences sur la santé humaine. On remarque que les élèves se soucient particulièrement des impacts directs de la pollution sur leur bien-être. Plusieurs reconnaissent qu'il s'agit là d'une motivation pour agir. En outre, le travail en équipe semble être un élément positif pour susciter leur intérêt. Le fait de prendre part à un projet structuré qui supporte les élèves dans leur démarche de résolution de problèmes semble constituer en soi une aide à l'action. En somme, après 6 semaines, les élèves ont modifié leurs conceptions du problème traité.

\section{Étape Planifier l'action}

La seconde partie de la démarche concernait la planification de l'action. Cette étape s'est déroulée entre les semaines 7 et 10. Il faut noter d'abord que les apprenants ont réussi à proposer une variété de solutions. Cependant, certaines solutions proposées étaient irréalisables faute de moyens ou en raison des caractéristiques du contexte guinéen. De plus, les apprenants ont fait généralement preuve de peu d'originalité dans leur formulation de solutions. Dans les trois écoles, la sensibilisation des populations a été proposée comme une des solutions possibles, faisant ainsi référence à l'idée de responsabilisation.

Tous les apprenants ont accepté de passer à l'action. Les raisons principalement évoquées par les jeunes sont l'état de leur milieu, les impacts sur la santé humaine et le désir d'aider les populations à mieux vivre. Ces raisons rappellent celles évoquées comme motivation pour l'action à la semaine 6 .

\section{Étape Mettre en œuvre et évaluer l'action}

La troisième partie de la démarche concernait le passage à l'action et l'évaluation de celle-ci. Les semaines 11 à 14 étaient prévues pour la mise en œuvre du plan d'action et la semaine 15 pour faire un retour sur la démarche vécue. La source d'information principale pour l'analyse de cette étape provient des questionnaires de la semaine 15. Il nous faut souligner ici que la validité des données recueillies par cet instrument est limitée. En effet, outre le fait qu'il s'agit, comme pour le questionnaire de la semaine 6, d'une auto-évaluation des apprenants transmettant leur perception de l'expérience vécue, il est à noter que plusieurs questions semblent avoir été mal comprises ou alors que la réponse fournie par les apprenants était confuse. Cela peut s'expliquer en grande partie par leur maîtrise généralement assez faible du français qui n'est pas leur langue maternelle, ni leur langue d'usage quotidien hors de l'école.

31 Les trois classes ont décidé de passer à l'action, malgré des délais importants, comme une grève générale et plusieurs difficultés organisationnelles rapportées par les 
assistants de recherche. On constate que $61 \%$ des jeunes ont estimé qu'aucun obstacle majeur n'avait nui à l'application de leurs solutions, ce qui peut avoir aidé à conserver leur motivation à cette étape. De plus, $89 \%$ des jeunes ont répondu qu'ils pensaient que les solutions retenues allaient avoir un impact à long terme dans leur milieu. Il peut sembler peu réaliste de croire à un impact à long terme des solutions appliquées, compte tenu de leur nature plutôt restreinte par rapport à l'ampleur des problèmes de l'eau à Conakry. Cependant, cela peut fournir un indice que la démarche vécue aura un effet sur leur engagement futur face à cet enjeu. D'ailleurs, $67 \%$ des apprenants ont dit envisager faire un suivi des actions entreprises.

$\mathrm{Au}$ terme de la démarche, les jeunes ont proposé quelques améliorations pertinentes. Beaucoup d'entre eux suggèrent de consacrer plus de temps à la sensibilisation de la population locale. Plusieurs expriment le souhait que le nombre de sorties sur le terrain soit augmenté et que plus de documentation leur soit accessible. Enfin, une diminution de la durée de la démarche est proposée par une majorité des participants des trois écoles; cette suggestion n'est pas étonnante pour les assistants de recherche sur le terrain qui ont observé une certaine lassitude s'installer dans les semaines précédant le passage à l'action.

\section{La démarche vécue dans chaque école}

\section{L'école Hamdallaye}

Il s'agit d'une classe de terminale, la dernière année du secondaire, en option sciences expérimentales. Ces jeunes sont plus âgés et ont plus de connaissances scientifiques ainsi qu'une meilleure maîtrise $d u$ français que les élèves du primaire de l'école Les Élites. On observe d'ailleurs, au cours de la première étape, qu'ils avancent plus que les autres groupes dans leur compréhension de la problématique et que leur manière de poser le problème se raffine. De plus, les solutions qu'ils proposent sont relativement plus variées et originales et leur texte de sensibilisation beaucoup plus complet. Néanmoins, on y retrouve, comme chez les Élites, certaines conceptions erronées qui demeurent jusqu'à la fin de la démarche au sujet de la transmission de maladies comme le paludisme et de la pollution de l'eau.

Par ailleurs, Hamdallaye est le seul groupe à avoir réussi à définir des critères adéquats pour l'évaluation des solutions proposées. Cependant, les élèves ne semblent pas les avoir appliqués correctement, car certaines solutions retenues sont inadéquates et d'autres sont irréalisables par les jeunes eux-mêmes. En outre, les élèves de terminale ont montré un intérêt marqué pour les sujets traités pendant la phase de construction du problème. Cet intérêt s'est traduit par les nombreux questionnements qu'ils ont exprimés et la qualité des discussions. L'enseignant a tout de même noté une baisse graduelle de leur intérêt vers la neuvième semaine. Cela peut s'expliquer par la longueur de la démarche. Un des points négatifs émis par les élèves de terminale est d'ailleurs la surcharge de travail et les heures supplémentaires exigées par la recherche. On peut penser qu'une stratégie possible pour conserver l'intérêt des élèves, ceux d'Hamdallaye comme ceux des Élites, aurait été de permettre aux apprenants une plus grande créativité dans l'élaboration de leur activité de sensibilisation, plutôt que de leur imposer une approche très formelle comme l'ont fait les enseignants. 


\section{L'école Les Élites}

35 La classe de 6e année de l'école Les Élites constituait le groupe le plus jeune de cette étude (11-12 ans). Les données révèlent que ces jeunes ont éprouvé des difficultés au niveau de la compréhension des consignes pour la recherche de solutions et la rédaction du plan d'action. On note également qu'ils restent campés tout au long de la démarche dans leur manière de poser et de résoudre le problème, sous forme d'interdits plutôt que de description d'une situation souhaitée. Le questionnement très directif de l'enseignant peut avoir contribué à cette étroitesse de la problématisation. Stoyanva (2000) explique à ce sujet que le questionnement de l'enseignant est très important pour aider les élèves à mieux poser un problème.

Certaines connaissances erronées exprimées au stade de la planification de l'action sont demeurées jusqu'à l'étape de passage à l'action. Ainsi, les textes de sensibilisation affirment que le paludisme est transmis par la pollution.

Concernant l'engagement de ces élèves, on observe que ceux-ci ont démontré un enthousiasme marqué au début de la recherche. Il faut dire que le scénario pédagogique proposé dans le cadre du projet de recherche, et particulièrement la sortie dans le milieu, constitue une nouveauté par rapport à la structure habituelle des leçons données à l'école. On a observé qu'ils avaient beaucoup apprécié cette sortie et on remarque aussi qu'ils ont été très stimulés par l'activité de sensibilisation des autres élèves de l'école. Par ailleurs, on a pu constater qu'ils n'expriment pas leur responsabilité personnelle dans la préservation de l'environnement et semblent plutôt s'en remettre aux autres; cela se reflète dans la quantité de solutions proposées qu'ils ne peuvent pas mettre en œuvre eux-mêmes.

\section{Le Centre de formation professionnelle de Donka}

Le groupe de jeunes adultes du CFP de Donka était formé d'étudiants en plomberie. Ces jeunes ont démontré qu'ils possédaient très peu de connaissances pour décrire la situation problème. Ils ont aussi exprimé beaucoup de difficultés à comprendre les étapes de la démarche et les consignes des différentes activités. La problématique étudiée a été la contamination de l'eau par les déchets humains, une appellation utilisée par ces jeunes pour désigner les excréments humains. Ils ne semblent pas être parvenus à dépasser leurs perceptions sensorielles du problème pour l'approfondir.

Ils ont tout de même proposé une variété de solutions, qui concernaient principalement l'entretien du réseau d'alimentation d'eau et l'assainissement. La plupart de ces solutions nécessitaient d'être mises en œuvre par d'autres acteurs qu'eux-mêmes. Il est à noter également que la sensibilisation a été mentionnée comme solution possible, mais n'a pas été retenue. Étant donné les difficultés d'expression orale et écrite de leurs étudiants, les enseignants ont préféré les diriger vers la mise en œuvre d'une autre solution, plus adaptée à leurs compétences manuelles: le nettoyage des caniveaux.

Par ailleurs, il a semblé plus difficile de susciter l'engagement des étudiants de Donka que dans les autres classes. Ces jeunes orientés vers un métier manuel ont semblé moins intéressés par le travail de réflexion proposé par la résolution de problèmes. Tout au long du processus, le taux d'absentéisme est demeuré élevé, ce qui a compliqué le suivi de la démarche. Ils ont tout de même exprimé différentes motivations pour s'engager et passer à l'action, la principale étant la propreté de leur milieu de vie. 
41 Selon leurs réponses au questionnaire de la semaine 15, il semble que les jeunes en formation considèrent la conservation de l'eau comme une responsabilité commune à tous. Cependant, l'incohérence de plusieurs réponses ainsi que des observations rapportées par les assistants de recherche laissent croire qu'ils ont complété le questionnaire rapidement, sans trop y porter attention. En fin de compte, c'est essentiellement leur engagement réel dans l'action de nettoyage qui témoigne de leur responsabilisation.

\section{Conclusions au regard de l'atteinte des objectifs}

Concernant la mobilisation des connaissances, la différence principale entre les démarches vécues dans les trois écoles a pu être observée lors de la première étape Poser le problème. Les élèves du primaire et ceux de la formation professionnelle sont demeurés à un niveau de définition du problème très immédiat, contenant peu d'informations et fondé sur leurs perceptions sensorielles. Leur façon de poser le problème n'a pas évolué beaucoup, alors que les élèves du secondaire ont réussi à élargir leur réflexion et leurs connaissances à cette étape.

Concernant l'engagement et l'intérêt des apprenants, le scénario proposé a semblé consacrer trop de temps à l'approfondissement de la compréhension du problème avant le passage à l'action. En effet, après un certain temps, les élèves des trois groupes ont exprimé une certaine lassitude, alors qu'un réel enthousiasme était observable lors des sorties d'exploration et du passage à l'action. Il pourrait donc être souhaitable de modifier le scénario pour l'orienter vers une démarche d'apprentissage expérientiel, dans laquelle les moments de réflexion, comme ceux consacrés à poser le problème, ne sont pas définis comme préalables à l'action, mais plutôt comme s'inscrivant dans un cycle continuel d'action-réflexion. L'action est alors conçue comme source de compréhension et de construction de sens et non seulement comme application d'une compréhension construite dans la réflexion. Dans une telle démarche, l'exploration active du milieu et la mise à l'essai de certains éléments de solution surviennent plus tôt. Le retour sur ces expériences permet d'acquérir des connaissances sur le problème, qui est en quelque sorte continuellement reconstruit par les élèves.

Enfin, concernant l'objectif de donner aux apprenants le désir d'agir, on constate que chaque classe a fait le choix de poursuivre jusqu'à l'action, malgré des conditions exigeantes, notamment dû au caractère inhabituel de ce type de démarche en milieu scolaire guinéen. Par ailleurs, on note également que les élèves n'ont pas pu constater les répercussions de leurs actions sur leur communauté et sur leur milieu immédiat. En effet, une fois le scénario complété dans le cadre du projet de recherche, aucune classe n'a poursuivi. Il est difficile donc d'affirmer qu'un désir durable de passer à l'action pour protéger et améliorer leur milieu de vie a été une conséquence de la participation de ces élèves à une démarche de résolution de problèmes. 


\section{BIBLIOGRAPHIE}

Bah, M. B. (2012). Apports, limites et enjeux de l'apprentissage par problèmes pour le développement de compétences associées au champ de l'éducation relative à l'environnement: une étude de cas en milieu universitaire guinéen. Thèse de doctorat inédite, Université du Québec à Montréal. Récupérée sur : http://www.archipel.uqam.ca/5267

Bardwell, L.V. (1991). Problem framing: A perspective on environmental problem-solving. Environmental Management 15, 603-612.

Bardwell, L.V., Monroe, M., Tudor, M. (1994). Environmental Problem Solving : Theory, Practice and Possibilities in Environmental Education. Troy (OH) : North American Association for Environmental Education.

Barrows, H. S. (1986). A Taxonomy of Problem-Based Learning Methods. Medical Education, 20, 481-486.

Boutet, M. (2002). Rapport final de l'intervention d'appui à l'ENSET de Matoto. Rapport d'une intervention d'appui à la mise sur pied d'une école normale d'enseignement technique et professionnel en République de Guinée, dans le cadre du projet AMORÉ-ETFP.

Camara, A. Y. (2004). SOS pour la Basse Guinée : Manque d'eau potable. PlaneteBleue.Info, Portail alternatif sur l'eau.

Chawla, L. (2002). Growing up in an urbanising world. London : Earthscan et UNESCO.

CIA (Central Intelligence Agency). (2009). The World Factbook, Guinea. Récupéré le 3 mars 2009 : www.cia.gov/library/publications/the-world-factbook/geos/gv.html

Commission Européenne Résidente en Guinée. (2007). Profil environnemental de la Guinée. Document de travail de base, 8 pages.

Donnay, J. et Cheffert, J. L. (1998). L'apprentissage par problème dans la formation initiale d'enseignants à l'Université. Liège : Presses de l'Université de Liège.

Driskell, D. (2002). Creating better cities with children and youth : A manuel for participation. London : Earthscan et UNESCO.

Éducation, formation professionnelle et jeunesse Manitoba (2001). L'éducation pour un avenir viable - Guide pour la conception des programmes d'études, l'enseignement et l'administration. Manitoba : Gouvernement du Manitoba.

Galaise, C. (2001). Approche pédagogique d'apprentissage par problèmes et connaissances conditionnelles en expertise comptable au premier cycle universitaire. Thèse de doctorat inédite, Université du Québec à Montréal.

Groupe de la Banque africaine de développement - Guinée (2009). Récupéré le 6 mai 2009 : www.afdb.org/fr/countries/west-africa/guInea/

Hart, R. (1997). Children's participation. London : Earthscan.

Jickling, B. (1992). Why I don't want my children to be educated for sustainable development. Journal of Environmental Education, 23(4) 5-8.

Ménard, L. (2007). Apprentissage par problèmes. Dans C. Raby et S. Viola, (Dir.). Modèles d'enseignement et théories d'apprentissage: De la pratique à la théorie (p 90-106). Montréal : Éditions CEC. 
Merriam, S.B. (1988). Case study research in education : A qualitative approach. San Francisco (CA) : Jossey-Bass.

Miles, M.B. et Huberman, A.M. (1991). Analyse de données qualitatives: recueil de nouvelles données. Bruxelles : De Boeck.

Ministère des Affaires étrangères et européennes de la République française. (2009). Récupéré le 2 mars 2009 : www.diplomatie.gouv.fr/fr/pays-zones-geo_833/guinee_349/index.html

Mucchielli, A. (1996). Dictionnaire des méthodes qualitatives en sciences humaines et sociales. Paris : Armand Collin.

Ouellet, L. (1994). La résolution de problèmes : fiche pédagogique. Document de travail. Québec : Ministère de l'eÉducation, Ministère de l'Environnement et de la Faune, Ministère des Ressources naturelles.

Pólya, G. (1971). How to solve it: a new aspect of mathematical method. Princeton (NJ) : Princeton University Press.

Proulx, L. P. (1999). La résolution de problèmes en enseignement : Cadre référentiel et outils de formation. Paris : De Boeck et Larcier.

Pruneau, D., Chouinard, O., Arsenault, C. et Breau, N. (1999). An international education project aiming at the improvement of people's relationship with the environment. International Research in Geographical and Environmental Education, 8, 26-39.

Pruneau, D., Freiman, V., Barbier, P. Y., \& Langis, J. (2009). Helping young students to better pose an environmental problem. Applied Environmental Education and Communication, 8(2), 105-113.

Pruneau, D., Kerry, J. et Langis, J. (2013). Étude des compétences propices au soin et à la transformation de l'environnement. Vertigo, 13. Récupéré : https://vertigo.revues.org/13411

Sauvé, L. (1997). Pour une éducation relative à l'environnement. Montréal : Guérin.

Sauvé, L., Orellana, I., Qualman, S. et Dubé, S. (2001). L'éducation relative à l'environnement, École et communauté : une dynamique constructive. Montréal : Éditions Hurtubise HMH.

Stoyanva, E. (2000). Empowering students' problem solving via problem posing. Australian Mathematics Teacher, 25, 33-37.

Treffinger, D. J. (1995). Creative problem solving : Overview and Educational implications. Educational Psychology Review 7, 301-312.

Tremblay, M. B. (2009). La pédagogie par problèmes. Une clé en enseignement postsecondaire. Processus de résolution de problèmes, méthode des cas et apprentissage par problèmes. Montréal : Guérin.

UNESCO-PNUE. (1978) Conférence Intergouvernementale sur l'éducation relative à l'environnement, Tbilissi, 14-26 octobre 1977: Rapport final. Récupéré le 6 novembre 2008 : http://unesdoc.unesco.org/images/0003/000327/032763fo.pdf

UNICEF. (2007). La situation des enfants dans le monde 2008 : La survie de l'enfant. Récupéré le 2 mars 2009 : www.unicef.org/french/sowc08/docs/sowc08_fr.pdf

Wheatley, G. H. (1995). Problem solving from a constructivist perspective. Dans D. R. Lavoie (Dir.), Toward a cognitive-science perspective for scientific problem solving (p. 11-22). Colombus (OH): National Association for Research in Science Teaching. 


\section{NOTES DE FIN}

i. La situation politique en Guinée depuis 2008 a considérablement retardé par la suite le travail de collaboration pour la production de cet article.

ii. Il est à noter que le professeur du CFP Donka pouvait être considéré, à cause de sa formation, comme un expert dans la qualité de l'eau. Cependant, il n'y a aucun indice dans les observations rapportées portant à croire qu'il a mis ses connaissances au service des apprenants.

\section{RÉSUMÉS}

Cet article décrit l'impact de la mise en œuvre d'une démarche de résolution de problèmes environnementaux par trois groupes d'élèves de la République de Guinée. L'objectif poursuivi était de mieux comprendre les enjeux d'une telle démarche au regard d'un engagement citoyen des jeunes africains pour le développement durable. Une classe du primaire, une du secondaire et une autre de la formation professionnelle ont travaillé à poser et à formuler des solutions à la problématique de la qualité de l'eau dans la capitale Conakry. Les données recueillies ont permis de constater l'intérêt des élèves pour ces questions, particulièrement lorsqu'elles sont appréhendées par observation directe sur le terrain; leur désir de passer à l'action a été également manifeste. Les principales limites observées sont l'accessibilité restreinte à des ressources documentaires ainsi que le manque de formation des enseignants en éducation à l'environnement et au développement durable.

This article describes the impact of implementing an environmental problem solving approach by three groups of students from the Republic of Guinea. The objective was to better understand the issues stemming from such an approach with regard to citizen engagement of young Africans towards sustainable development. One classroom from elementary school, one form secondary school and one from a vocational school worked to lay down and formulate solutions to the problem of water quality in the country capital, Conakry. The data collected showed students' interests towards these questions, especially when they are apprehended by direct observation in the field; their desire to take action was also evident. The main limitations observed are the limited access to documentary resources as well as the lack of training of teachers in environmental and sustainable development education.

\section{INDEX}

Index géographique : République de Guinée

Keywords : environmental problem solving, environmental and sustainable development education, eco-citizenship

Mots-clés : résolution de problèmes environnementaux, éducation à l'environnement et au développement durable, écocitoyenneté

\section{AUTEURS}

\section{MARC BOUTET}

Marc Boutet est détenteur d'un doctorat en didactique de l'Université Laval (2000). Il enseigne et mène des recherches dans le domaine de la formation à la pratique de l'enseignement ainsi que dans le domaine de l'éducation relative à l'environnement et au développement durable. Il est 
président de l'Association pour la formation à l'enseignement (AFORME). Il est chercheur associé au CRIFPE (Centre de recherche interuniversitaire sur la formation et la profession enseignante).

\section{AMÉLIE GADBOIS}

Amélie Gadbois est bachelière en biologie de l'Université Laval et a complété une maîtrise en écologie internationale à l'Université de Sherbrooke. Ses expériences de travail, ainsi que ses études l'ont menée à approfondir sa réflexion sur l'éducation relative à l'environnement au cours d'un essai de fin d'étude. Elle a, de plus, cumulé de nombreuses expériences en interprétation de la nature auprès d'organismes à but non lucratif en collaboration avec Parcs Canada.

\section{GHISLAIN SAMSON}

Ghislain Samson, titulaire d'un doctorat (Ph. D.) en didactique des sciences au secondaire (UQTR/ UQAM), est professeur au Département des sciences de l'éducation de l'UQTR. Spécialiste de la didactique des sciences et du transfert des apprentissages, il s'intéresse également aux questions relatives au curriculum scolaire, à l'interdisciplinarité et à l'éducation relative à

l'environnement. Outre ses intérêts de recherche en éducation scientifique, il conduit des recherches touchant les élèves en difficulté, l'employabilité et l'insertion socioprofessionnelle des jeunes.

\section{MAMADOU BHOYE BAH}

Mamadou Bhoye Bah a obtenu son doctorat en éducation de l'UQAM. Il est enseignant chercheur au Centre d'étude et de recherche en environnement (CÉRE) de l'Université de Conakry, en Guinée. Membre du comité scientifique du Réseau international francophone de recherche en ERE de 2004 à 2012 (RefERE), M. Bah a conduit trois projets pédagogiques pilotes dans le domaine de l'ERE. Il a également présenté plusieurs communications ces dernières années à Montréal, Paris, Ouagadougou, Kigali et Bamako.

\section{KADIATOU NOUR DIALLO}

Kadiatou Nour Diallo est professeure à l'École normale des professeurs de l'enseignement technique et professionnel (ENPETP) de la République de Guinée. Après une formation de premier cycle en génie mécanique à l'Université de Conakry, elle a poursuivi des études de maîtrise en enseignement à l'Université de Sherbrooke de 2003 à 2005, avec une spécialité en intégration des dimensions environnementales dans la pratique pédagogique de l'ETFP. Elle a été la coordonnatrice terrain du projet. 